\title{
Vector analyzing powers of the deuteron-proton elastic scattering and breakup at $100 \mathrm{MeV}$
}

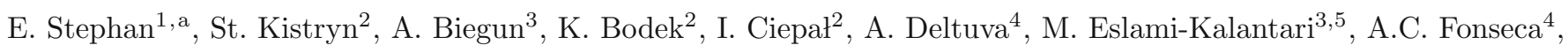
I. Gasparić ${ }^{3}$, J. Golak ${ }^{2}$, L. Joulaeizadeh ${ }^{3}$, N. Kalantar-Nayestanaki ${ }^{3}$, H. Kamada ${ }^{6}$, B. Kłos ${ }^{1}$, A. Kozela ${ }^{7}$, J.G. Messchendorp ${ }^{3}$, H. Moeini ${ }^{3}$, A. Nogga ${ }^{8}$, W. Parol ${ }^{2}$, A. Ramazani-Moghaddam-Arani ${ }^{3,9}$, R. Skibiński ${ }^{2}$, R. Sworst ${ }^{2}$, A. Wilczek ${ }^{1}$, H. Witała ${ }^{2}$, and J. Zejma ${ }^{2}$

1 Institute of Physics, University of Silesia, PL-40007 Katowice, Poland

2 Institute of Physics, Jagiellonian University, PL-30059 Kraków, Poland

3 Kernfysisch Versneller Instituut, NL-9747 AA Groningen, The Netherlands

4 Centro de Fisica Nuclear da Universidade de Lisboa, P-1649-003, Portugal

${ }^{5}$ Faculty of Physics, Yazd University, Yazd, Iran

6 Department of Physics, Kyushu Institute of Technology, 804-8550 Japan

7 Institute of Nuclear Physics, PAN, PL-31342 Kraków, Poland

8 Forschungszentrum Jülich, IKP-3 (Theorie), IAS-4 and JCHP, D-52425 Jülich, Germany

9 Faculty of Physics, University of Kashan, Kashan, Iran

Received: 11 July 2012 / Revised: 27 December 2012

Published online: 19 March 2013

(c) The Author(s) 2013. This article is published with open access at Springerlink.com

Communicated by Hayan Gao

\begin{abstract}
High-quality vector analyzing power data for the ${ }^{1} \mathrm{H}(\mathbf{d}, \mathrm{pp}) \mathrm{n}$ breakup reaction and elastic scattering at $100 \mathrm{MeV}$ beam energy have been measured in a large part of the phase space for these processes. The results are compared to theoretical predictions obtained using the charge-dependent Bonn potential alone or combined with the three-nucleon force TM99 as well as to the results of calculations in the coupled-channel approach, with or without the inclusion of the Coulomb interaction. In the studied observables, effects of the $3 \mathrm{NF}$ and the Coulomb force are almost absent. The pairwise NN interactions alone are sufficient to describe the experimental results.
\end{abstract}

\section{Introduction}

Thorough understanding of nuclear forces is one of the basic goals of nuclear physics. Most commonly, the interaction between nucleons is described within meson exchange theories or, alternatively, in the framework of the Chiral Perturbation Theory (ChPT). The predictions of both approaches can be reliably tested only in the simplest systems, for which rigorous, numerically exact calculations can be performed. Except for bound states, this condition is presently fulfilled only for systems of two or three nucleons. For four nucleons the rigorous calculations are still limited to the domain of low energies, below the energy threshold for the deuteron breakup [1,2]. A very rich set of experimental data for two nucleon (pp and $n p$ ) systems, covering a wide range of energies, was analyzed and parametrized in terms of phase shifts [3]. It has been demonstrated that both, realistic potentials and potentials derived from ChPT, could very precisely describe all the

\footnotetext{
a e-mail: elzbieta.stephan@us.edu.pl
}

data for the $2 \mathrm{~N}$ systems. When the studies are extended to systems of three nucleons, the question arises whether the pairwise interactions provide the only contribution to the dynamics of the system. Additional contributions, socalled three-nucleon forces (3NFs), arise naturally in the framework of ChPT [4]. Also in the meson exchange picture, a $3 \mathrm{~N}$ interaction can be derived by means of two-pion exchange between all three nucleons with an intermediate excitation of one of them to a virtual $\Delta$ state. This so-called Fujita-Miyazava force [5] was a starting point for the modern ways of modelling 3NFs, like TM99 [6], Brazilian [7], Urbana IX [8] or Illinois [9].

A first indication of the significance of $3 \mathrm{NF}$ contributions came from the ${ }^{3} \mathrm{H}$ and ${ }^{3} \mathrm{He}$ bound state studies [10]. Further evidences on the important role of $3 \mathrm{NFs}$ were deduced from the nucleon-deuteron elastic scattering observables $[11,12]$. The data base for the elastic nucleondeuteron scattering has been recently enriched by a large number of precise experiments performed in various laboratories [13-27]. The results of those studies confirm the importance of $3 \mathrm{NFs}$ for describing the elastic scattering 
process at medium energies, but demonstrate also certain deficiencies of the present $3 \mathrm{NF}$ models. The emerging picture is rather complex, especially in the sector of polarization observables.

Studies of the nucleon-deuteron breakup process are another important source of information on the $3 \mathrm{~N}$ system and, due to the kinematical variety of final states, can shed some light on the kinematical dependencies of $3 \mathrm{NF}$ effects, and possibly also on their dynamical origin. At present, breakup observables can be predicted rigorously via exact solutions of the Faddeev equations with nuclear forces modelled in various ways. At intermediate energies, the most commonly used are realistic NN potentials combined with model $3 \mathrm{~N}$ forces [11], two- and threenucleon interactions obtained by an explicit treatment of the $\Delta$-isobar excitation within the coupled-channel potential (CCP) framework [28-31] or dynamics generated on the basis of the chiral perturbation theory at the nextto-next-to-leading order [32] with all relevant $\mathrm{NN}$ and $3 \mathrm{~N}$ contributions taken into account. The predicted relative contribution of $3 \mathrm{~N}$ effects rises with the beam energy and also depends strongly on the phase space region and the observable under study.

Following the studies of the ${ }^{2} \mathrm{H}(\mathbf{p}, \mathrm{pp}) \mathrm{n}$ reaction at $65 \mathrm{MeV}$, performed for selected geometries [33-38], the first investigations of the ${ }^{1} \mathrm{H}(\mathbf{d}, \mathrm{pp}) \mathrm{n}$ reaction over a large phase space region were performed at an equivalent deuteron beam energy of $130 \mathrm{MeV}$. Precision of the experimental cross section data and coverage of a large fraction of the phase space allowed those experiments [39-41] to reliably test predictions of various theoretical approaches. The investigations revealed both, sizable 3NF effects [39, 40], as well as significant influences of the Coulomb interaction [41]. In contrast, studies of polarization observables have shown that vector analyzing powers at that energy were very well described by pairwise NN interactions alone $[42,43]$. Tensor analyzing powers were also well reproduced by calculations in almost the whole studied region, only locally certain discrepancies have been observed $[42,44]$. For $A_{x y}$ such discrepancies appeared or were enhanced when model 3N forces, like TM99 or Urbana IX, were included.

Studies of polarization observables for the breakup reaction were recently performed also at higher energies. Certain problems with describing (proton) vector analyzing powers were demonstrated by the data measured for the p-d breakup at $135 \mathrm{MeV}$ [45] and $190 \mathrm{MeV}$ [46]. Vector (deuteron) analyzing power and spin correlation coefficients for the d-p breakup at $270 \mathrm{MeV}(135 \mathrm{MeV} /$ nucleon $)$ are relatively well described by pure NN interactions, while the remaining discrepancies are not removed by adding $3 \mathrm{NF}$ [47]. A measurement of several configurations of the d-p breakup data at $135 \mathrm{MeV} /$ nucleon [48] demonstrates large $3 \mathrm{NF}$ effects for tensor analyzing powers and transfer coefficients, however, none of the model $3 \mathrm{NFs}$ is able to describe correctly all these observables. The deuteron vector analyzing powers at that energy do not reveal, in contrast to the theoretical predictions, any effect of 3NF. The inclusive experiments of p-d breakup at $248 \mathrm{MeV}$ suggest problems with description of the differen- tial cross section [49], recently confirmed by the inclusive measurement at $250 \mathrm{MeV}$ [50]. An extensive discussion of the present status of understanding of the $3 \mathrm{~N}$ system dynamics can be found in recent reviews [51,52].

Studies at high energies are strongly motivated by the fact that the predicted relative contribution of the $3 \mathrm{NF}$ rises with an energy. However, in view of the results mentioned above, such predictions are not necessarily confirmed by the data. Moreover, possible quantitative conclusions on the magnitude of the $3 \mathrm{NF}$ effects can be verified, provided that in the region where the predictions for these effects are negligible or absent, the NN interactions alone do describe the data well. Therefore, the experimental data that cover a large range of energies, including also relatively low ones, are important for systematic tests of theoretical predictions and for understanding of possibly arising discrepancies. The domain of relatively low energies can be also of particular interest for testing predictions of ChPT. These considerations motivated measurements of $3 \mathrm{~N}$ system observables with a polarized deuteron beam of $100 \mathrm{MeV}$. The present paper is devoted to the vector analyzing power data obtained for the $\mathbf{d}+{ }^{1} \mathrm{H}$ elastic scattering and for the ${ }^{1} \mathrm{H}(\mathbf{d}, \mathrm{pp}) \mathrm{n}$ breakup reaction. The available range of kinematical configurations of the breakup reaction was divided into about 400 data points.

The experimental setup is described in sect. 2. Section 3 outlines the data analysis procedures: event selection, energy calibration, determination of the beam polarization and, finally, evaluation of the vector analyzing powers for the elastic scattering and for the breakup reactions. Results obtained for the vector analyzing power $i T_{11}$ of the elastic scattering are shown and the experimental uncertainties of all the results are discussed. The $A_{x}$ and $A_{y}$ data obtained for the breakup reaction and their comparison with the theoretical calculations are presented in sect. 4 and the conclusions are given in sect. 5 .

\section{Experimental setup}

The experiment was carried out at the Kernfysish Versneller Instituut (KVI) in Groningen, The Netherlands, using a deuteron beam accelerated to an energy of $100 \mathrm{MeV}$ in the AGOR cyclotron. A beam of deuterons, vector and tensor polarized in the transversal direction, was produced in an atomic-beam type polarized ion source, POLIS [53]. POLIS proved to be very stable in terms of current and of polarization magnitude.

Five polarization states were used, described by the maximal vector and tensor polarization values $P \equiv$ $\left(P_{Z}, P_{Z Z}\right):(0,0),\left(+\frac{2}{3}, 0\right),\left(-\frac{2}{3}, 0\right),(0,-2),(0,+1)$. These values correspond to $100 \%$ efficient transitions between the states of atomic deuterium. The beam polarization states were changed sequentially in a programmed cycle. Each state was set for five minutes, while switching between the subsequent states took a few seconds.

The polarized deuteron beam was guided through a beam line to the experimental setup and focused on the liquid-hydrogen target [54]. It was further transported to the Faraday Cup, where the beam current of about $10 \mathrm{pA}$ 


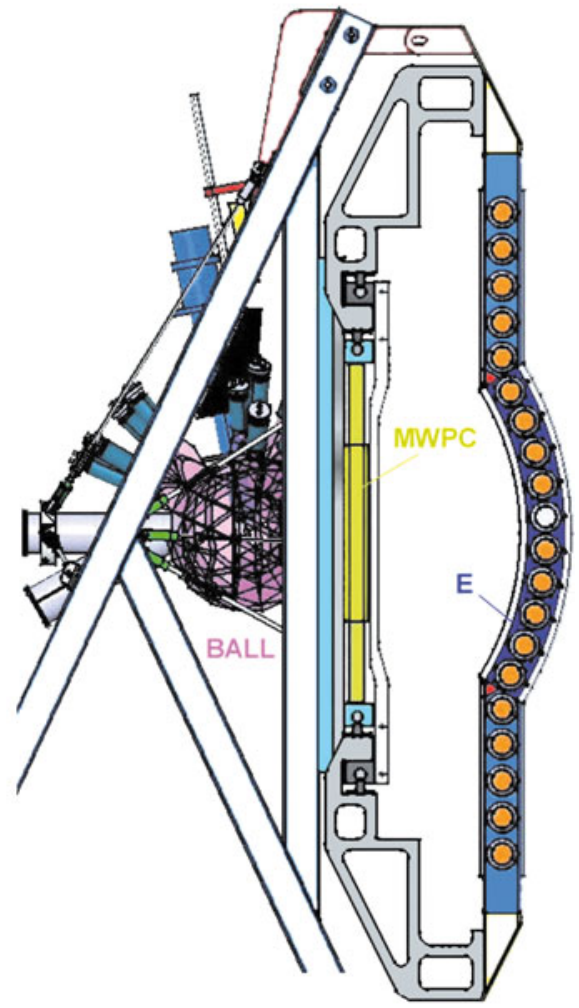

Fig. 1. Schematic view of the BINA detector. The beam enters the setup from the left, a target is positioned at the center of the Ball sphere. The MultiWire Proportional Chamber and the arc of $E$ hodoscope are shown.

was measured. The charged reaction products were detected in BINA, Big Instrument for Nuclear Polarization Analysis [27], see fig. 1. The BINA detection system is built of two parts: Wall (forward) and Ball (central and backward). The Wall, resembling the former SALAD system [55], consists of a MultiWire Proportional Chamber (MWPC) [56] and an $E$ hodoscope, for the determination of the emission angles and energies of the charged reaction products, respectively. The setup can be also equipped with a $\Delta E$ (transmission) hodoscope, positioned between those two elements. However, for the experiment at a relatively low beam energy, the $\Delta E$ detector has been dismounted in order to reduce the energy threshold for the registered particles. The Wall covers the polar angles $\theta$ between $10^{\circ}$ and $35^{\circ}$, and the full range of the azimuthal angle $\varphi$. MWPC has three planes with vertical, horizontal and diagonal wires, and is used for precise reconstruction of the particle emission angles, with the overall accuracy of $0.3^{\circ}$ for $\theta$ and between $0.6^{\circ}$ and $3.0^{\circ}$ for $\varphi$. The $E$ hodoscope is a set of ten horizontal scintillator bars, forming an arc with the center at the target position. The bars are $12 \mathrm{~cm}$ thick and over two meters long. Each of them is read out by two photomultiplier tubes, mounted on both ends of the bar. The central bars are partially cut to form a hole for the beam pipe. The Ball is a set of 149 phoswitch detectors making together a part of a sphere, covering polar angles between $40^{\circ}$ and $160^{\circ}$ (with an opening for the target holder). The Ball forms itself the vacuum-tight scattering chamber, with the target placed at the center of the sphere, therefore the energy loss of particles on their way from the target to detectors is minimized. The Ball is made of scintillator prisms composed of a "fast" plastic scintillator BC408 with a $1 \mathrm{~mm}$ thin "slow" scintillator (BC-444) layer glued on its face. The thickness of the "fast" part depends on the detector position: it is $90 \mathrm{~mm}$ for polar angles smaller than $100^{\circ}$ and $30 \mathrm{~mm}$ at larger angles (not used in measurements in the d-p kinematics).

For BINA, the electronic, read-out and data acquisition systems of the former SALAD setup [42] were adapted. The trigger conditions were based on hit multiplicities in three groups of photomultiplier tubes (PMTs): left-side PMTs of the Wall, right-side PMTs of the Wall and all PMTs of the Ball. Three types of events were registered, with separately downscaled rates: coincidences of two particles registered in the Wall (Wall-Wall coincidences), events with at least one particle registered in the Wall and at least one in the Ball (Wall-Ball coincidences) and, strongly suppressed by means of downscaling, events with at least one particle registered in the whole setup (single events). An easy way of remote choice of triggers was applied, based on logic units and Output Registers. Control over the read-out was performed by the FERA system. Details on a sectioned FERA system with distributed gates, realized with the use of Tagger and Extender modules, can be found in ref. [57]. Changes in the read-out as compared to the SALAD system were mostly related to a substantial increase of the number of detector channels and to differences in pulse shapes generated in different detector parts. The modules to be read out were the TDC's of the Wall, the ADC's of Wall and Ball, and PCOS of MWPC. Separate ADC gates had to be constructed for the Wall hodoscope and for the Ball part; for the latter, gates of two different lengths were used for integrating signals of the Ball phoswitches. For these reasons a total number of FERA subsections had to be increased. The front-end processor, RIO3 with the LynxOS real-time operating system, controlled the data acquisition with the use of Multi-Branch System (MBS) [58]. The processor was hosted in a VME crate, together with the buffer memories, two Lecroy LC1190 modules, and the socalled Trigger module. The handshaking was realized by the strobe/acknowledge asynchronous protocol between the module currently controlling the bus (ADC, TDC or PCOS) and the memory (mediated by the FERA Drivers): For each data word to be sent from the FERA system to the memory, a strobe signal was produced for the purpose of synchronization, and the memory confirmed receiving the data by sending an acknowledge signal. This mechanism featured also a simple method of avoiding a memory overflow: Strobes were counted in an external downcounter module and when their number reached the programmed level, corresponding to an almost full memory buffer, a signal was generated by the downcounter, causing a processor interrupt. As a reaction to the interrupt, the processor disabled this memory and enabled the other one. Only then it read out the full memory content, directed the data to 

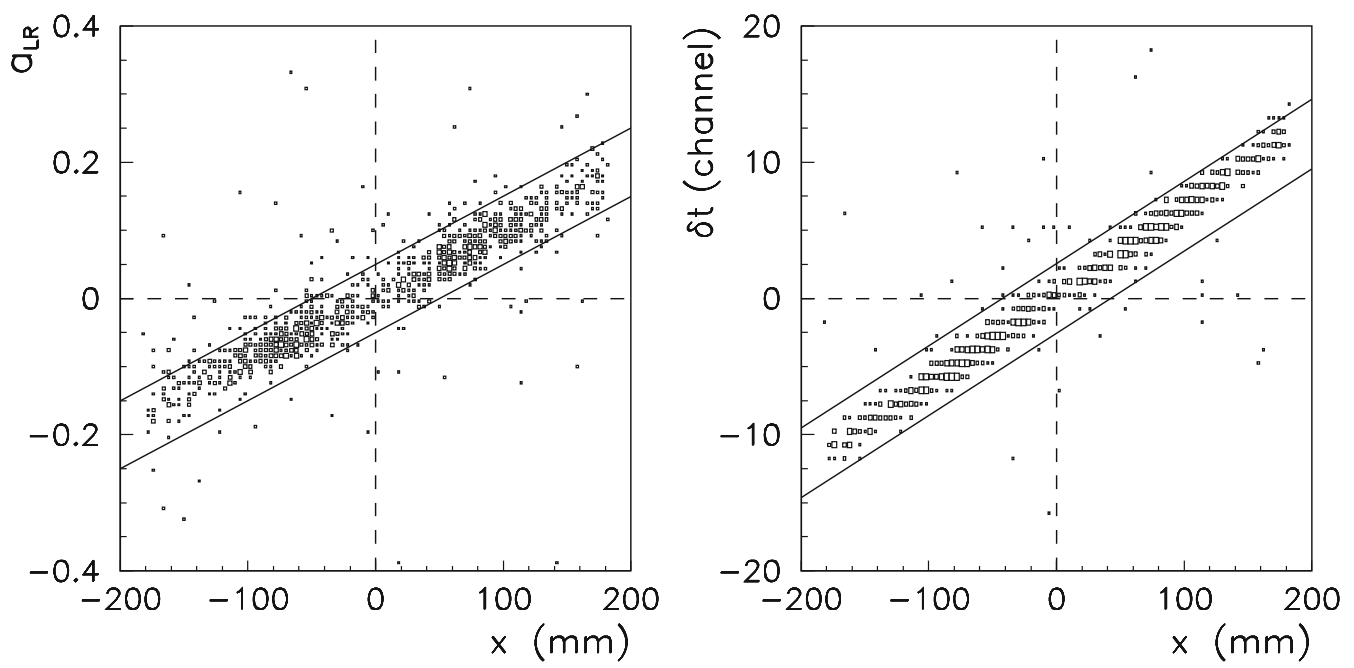

Fig. 2. Examples of the position correlation of signals from one scintillator bar of the $E$ hodoscope with hits in the MWPC: Asymmetry $a_{L R}$ and time difference $\delta t$ are plotted versus horizontal position reconstructed from hits in the $x$-plane of the MWPC. The spectra were obtained under the condition that the vertical position reconstructed from the MWPC corresponded to the geometrical acceptance of that $E$ detector. Oblique lines represent limits set to select correctly correlated events.

an archive disk and sent a fraction of the data stream to the on-line analysis for monitoring purposes.

\section{Data analysis}

\subsection{Events selection and energy calibration}

The events of interest were coincidences of two charged particles, i.e. proton-proton pairs from the breakup process or deuteron-proton pairs from the elastic scattering. In this work only kinematical configurations of the breakup reaction leading to Wall-Wall coincidences are shown. The elastic scattering events of two types are considered: Wall-Wall and Wall-Ball coincidences.

The first step of the analysis consisted of selecting particles which were produced within a single beam burst. Among the particles registered in the Wall, only the hits appearing within a $20 \mathrm{~ns}$ time window were accepted. Time information was not available for particles registered in the Ball detectors, therefore all of them were accepted. In the second step, the position correlation of the hits in the Wall scintillators with the hits in MWPC was established. Such correlation is usually straightforward with the exception of certain events when, for example, an extra hit: a neutron, detector noise or a background particle, was registered in the Wall, or the distance between the two proton hits, measured along one of the coordinates, was small. Therefore, to avoid ambiguity, it was favourable to make use of at least approximate position information, both vertical and horizontal, from the hodoscope. In the BINA experimental setup (without a $\Delta E$ hodoscope) the scintillator is segmented in the vertical direction. Information on the position along the scintillator bar can be found by comparing signals from the two PMTs mounted on both ends of the given $E$ bar. The position of the particle hit along the bar is reflected in an asymmetry of pulse heights $C_{L}, C_{R}$ of two PMTs and also in time difference $\delta t=t_{L}-t_{R}$ of registering the signals in those PMTs. Both these dependencies were used. The asymmetry,

$$
a_{L R}=\frac{C_{L}-C_{R}}{C_{L}+C_{R}},
$$

and the time difference, $\delta t$, were compared with the $x$ position reconstructed from the MWPC information. Examples of $a_{L R}$ and $\delta t$ versus $x$ spectra for one detector are shown in fig. 2, together with the lines limiting the ranges of the accepted events. For almost all events a very clear correlation of position information from the hodoscope and from the MWPC is observed, confirming that hits in these two parts of the detector belong to the same track of one charged particle. The remaining events, which may correspond to the accidental correlation between MWPC and a hodoscope element, caused by noise, background or charged particles scattered in the detector material, were rejected from further analyses.

For the purpose of energy calibration, elastic scattering events were measured during special runs with energy degraders (steel plates) positioned in front of the scintillation hodoscope. The position of the peak corresponding to protons from the elastic scattering which traversed the degraders was compared with the result of simulations taking into account all their energy losses. In the first step a linear function was fitted to the relation of the calculated deposited energy versus the measured pulse height. For the BINA Wall elements a significant light attenuation was observed. In order to suppress its main, exponential component, a geometric average $\sqrt{C_{L} C_{R}}$ was used instead of pulse heights of individual PMTs, $C_{L}$ and $C_{R}$, or their sum. The remaining, weak dependence of such average on the hit position was introduced into the calibration parameters.

When charged particles deposit their energy in the plastic scintillator, the light quenching in the scintillating 

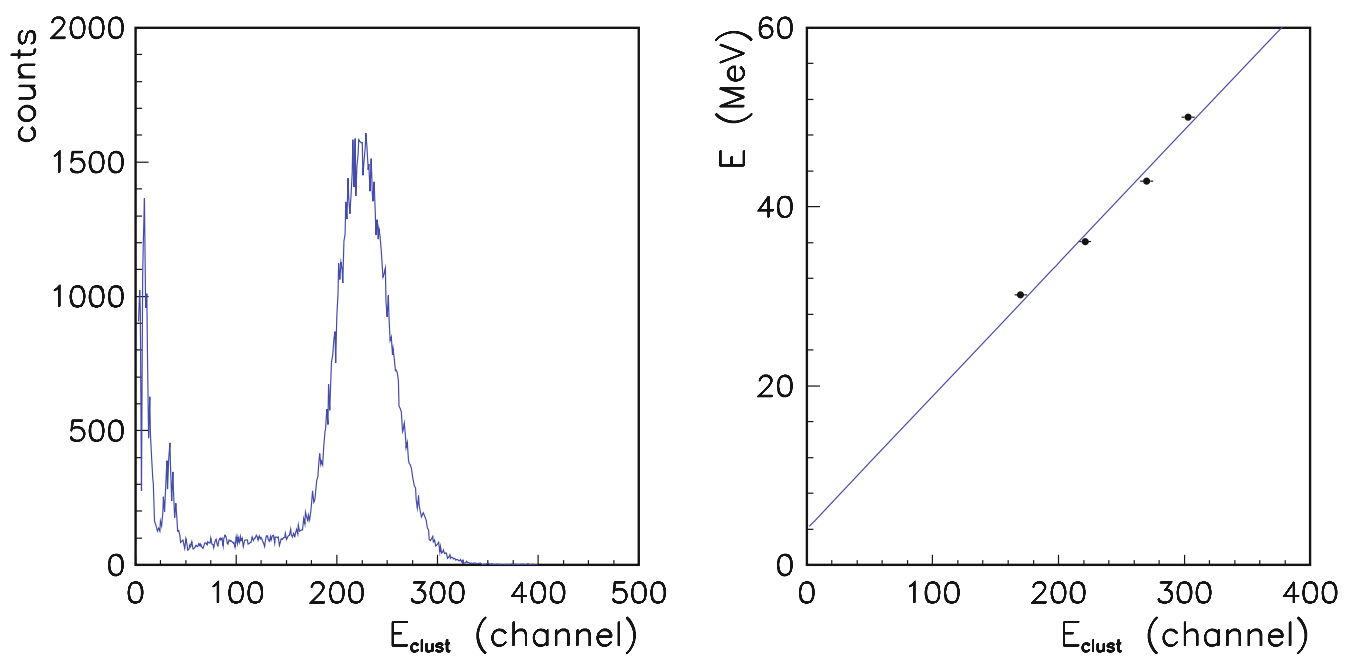

Fig. 3. Left: An example of the energy spectrum of clusters centered (the highest pulse) in one chosen Ball element; only particles coplanar to the deuterons registered in the Wall at $\theta_{d}^{l a b}=24^{\circ} \pm 0.5^{\circ}$ are selected. Right: Energy calibration of that detector obtained as a relation between the positions of the peaks corresponding to four angles of the coincident deuteron and the known energy of the elastically scattered protons.

material causes non-linear dependence between the energy deposit and the height of the induced pulses. That effect can be described by the Birks formula [59], or by its modified form introduced by Chou [60]. For protons with energies of tens of $\mathrm{MeV}$ the non-linearity is small, therefore the effect was introduced to the calibration by a slight change of the slope of the linear dependence below $30 \mathrm{MeV}$. In the last step of the calibration, the relation between the energy deposited by protons in the $E$ counter and their energy at the moment of reaction was found by the Monte Carlo simulation of the energy loss.

The precise calibration of the Ball detectors was not required for the data set presented in this work, since the Wall-Ball coincidences were analyzed only for the purpose of reconstructing the elastic scattering events. In such case, the full kinematics can be obtained from the precisely measured angle of the deuteron, registered in the Wall. The identification of these events relied on simple cuts: a choice of deuteron polar angle $\theta_{d}$, requirement of coplanarity and, finally, a cut on the energy spectrum of particles registered in the Wall, constructed under the two previous conditions. The angles of deuteron emission were known precisely from the MWPC information, while the proton angle was reconstructed as corresponding to the geometrical center of the face of the responding Ball detector element. In order to select coplanar events, the difference of both azimuthal angles was required to be around $180^{\circ}$ with a tolerance equal to the maximal azimuthal acceptance of a Ball detector element. Due to the light leakage between Ball elements, usually more than one detector had registered a hit. In such case, for proton angle determination and gain matching, the element with the highest pulse was chosen. In the next step, the energy deposited (pulse height) in all detectors forming a cluster was summed up. An example of the resulting proton energy spectrum for $\theta_{d}^{l a b}=24 \pm 0.5^{\circ}$ is shown in fig. 3 , left panel. In spite of its large width, the proton peak can be well identified and the low-energy background can be efficiently rejected.
Each Ball element covers a few degrees of polar angle. By choosing particular $\theta_{d}$ angles for deuterons registered in the Wall, one can select certain polar angles of protons registered in the Ball element. Analyzing the obtained energy spectra and comparing them with the known proton energies, an approximate energy calibration of Ball detectors was performed, cf. fig. 3, right panel. This method can in future be applied for the determination of the energy of the breakup protons registered in the Ball. For the scope of this work, the obtained linear channel-energy relation confirms the correct selection of the elastic scattering events.

\subsection{Determination of the beam polarization}

In this work, we present results for vector analyzing powers, therefore only the two beam states with nominally pure vector polarization $P:\left(+\frac{2}{3}, 0\right),\left(-\frac{2}{3}, 0\right)$ and an unpolarized beam are of interest.

The procedure applied to determine values of the beam polarization was based on the analysis of the elastic scattering events. After normalization to the beam current and correction for the dead time, the rates of the elastic scattering events $N_{P}^{\theta}(\phi)$ for the selected polarization state $P$ and the polar angle of the deuteron $\left(\theta \equiv \theta_{c . m}^{d}\right.$. $)$ were obtained as a function of the angle $\phi$ defined according to the convention of ref. [61]. $N_{0}^{\theta}(\phi)$ denotes such a rate obtained for the state with an unpolarized beam. On the basis of these numbers a ratio,

$$
f_{P}^{\theta}(\phi)=\frac{N_{P}^{\theta}(\phi)-N_{0}^{\theta}(\phi)}{N_{0}^{\theta}(\phi)},
$$

was constructed.

The evaluated rates of events are directly proportional to the yield of scattering of transversally polarized spin-1 particles off an unpolarized target. According to formulae 


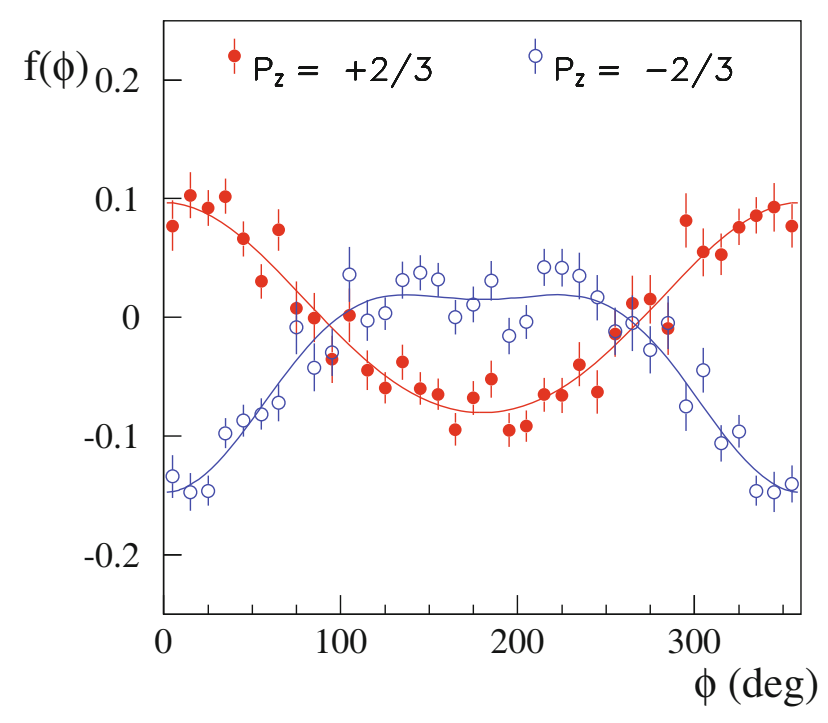

Fig. 4. Ratios $f_{P}^{\theta}(\phi)$ for two vector polarized states and one chosen polar angle $\theta_{d}^{\text {c.m. }}=129.03^{\circ}$ (symbols), together with lines resulting from the fit, as described in the text. The error bars represent statistical uncertainties. The nominal (maximal) vector polarization values are indicated in the panel.

in ref. [61], the ratio (2) can be expressed (in the spherical coordinates) as

$$
\begin{aligned}
f_{P}^{\theta}(\phi)= & i T_{11}^{e l}(\theta) \sqrt{3} P_{Z} \cos \phi \\
& -T_{22}^{e l}(\theta) \frac{\sqrt{3}}{2} P_{Z Z} \cos 2 \phi-T_{20}^{e l}(\theta) \frac{\sqrt{2}}{4} P_{Z Z},
\end{aligned}
$$

where $i T_{11}^{e l}(\theta)$ and $T_{22}^{e l}(\theta), T_{20}^{e l}(\theta)$ are spherical vector and tensor analyzing powers of the elastic scattering process. An example of the functions $a \cos \phi+b \cos 2 \phi+c$ fitted to distributions of the experimental ratio $f_{P}^{\theta}(\phi)$ is shown in fig. 4. The shapes of the distributions indicate that the state with positive vector polarization is almost pure ( $\cos \phi$ dependence strongly dominates), while in the other state also a certain contribution of tensor polarization is present.

An accurate knowledge of the analyzing powers is necessary for determining the vector and tensor polarizations from the fitted parameters. As mentioned before, we aim at determining $P_{Z}$ alone, so for this purpose the vector analyzing power $i T_{11}^{e l}$ is of interest. That observable has been measured at the deuteron beam energies of $95 \mathrm{MeV}$ and $106 \mathrm{MeV}$ [62]. Slow variation of $i T_{11}^{e l}$ with energy validates the use of a linear interpolation of these data to the energy of $100 \mathrm{MeV}$. The reference data obtained by such an interpolation are shown in fig. 5 as full dots. In order to improve statistical accuracy of the determined polarization value, the whole angular distribution has been used. Vector polarization $P_{Z}$ was determined for each of the two states in a way to achieve the best consistency (guaranteed by a $\chi^{2}$ criterion) of the corresponding $i T_{11}^{e l}$ distribution with that of the reference data (details of the method, applied to a different data set, can be found in ref. [63]). The results are shown in table 1 . Weighted averages of $i T_{11}^{e l}$ values obtained for both states are shown in fig. 5 together with

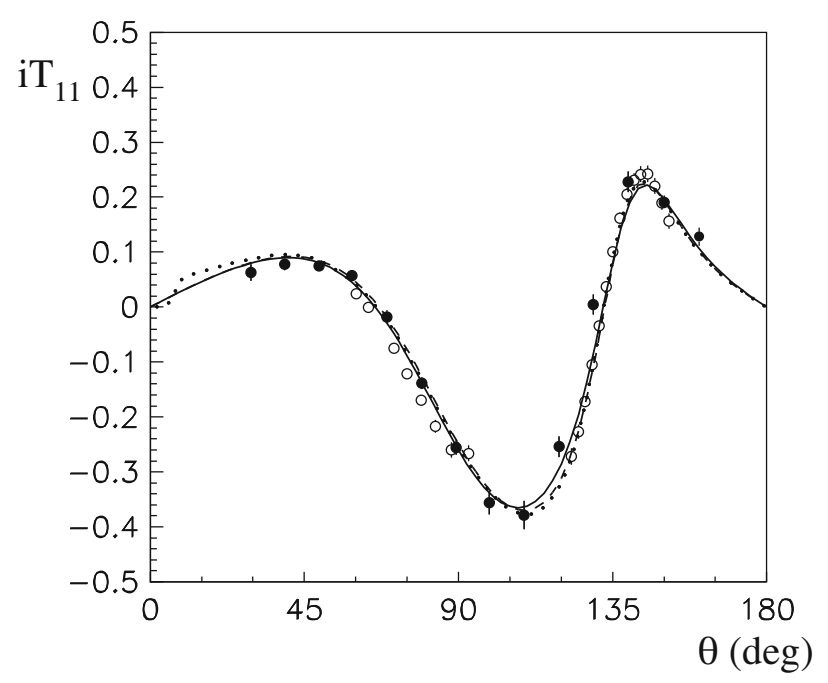

Fig. 5. Vector analyzing power of the elastic d-p scattering at $100 \mathrm{MeV}$ : empty dots - this work, full dots - the reference data from [62] after interpolation to the energy of $100 \mathrm{MeV}$ (see text). Theoretical predictions obtained with the CD-Bonn potential without and with TM99 3NF are shown as dashed and solid lines, respectively, while the dotted line represents CCP calculations with the Coulomb interaction included.

Table 1. Beam polarization values obtained for two "pure vector" beam states; for more details see text.

\begin{tabular}{|c|r|r|r|}
\hline$P$ & $P_{Z}$ & $\Delta P_{Z}^{\text {stat }}$ & $\Delta P_{Z}^{\text {sys }}$ \\
\hline$\left(-\frac{2}{3}, 0\right)$ & -0.475 & 0.024 & 0.025 \\
$\left(+\frac{2}{3}, 0\right)$ & 0.439 & 0.023 & 0.025 \\
\hline
\end{tabular}

the reference data. Good consistency of the shapes of the two distributions can be observed, with the exception of a small difference of slopes for polar angles between $120^{\circ}$ and $140^{\circ}$. Such a discrepancy can be due to systematic uncertainties of both data sets (e.g. related to absolute determination of angles or beam energies) and of the interpolation procedure. The resulting systematic uncertainties are estimated to be of about $5 \%$ for $P_{Z}$ (see table 1) and of 0.01 for $i T_{11}^{e l}$. The data are compared to predictions of the theoretical calculations with the CD-Bonn potential alone and combined with TM99 3NF, and also with the CCP calculations including Coulomb interaction [30,31]. All the curves representing individual calculations practically lie on top of each other, thus no effect of TM99 3NF nor of Coulomb interaction is predicted. The comparison to the experimental results shows that the CD-Bonn potential alone is sufficient to describe the data accurately.

\subsection{Evaluation of vector analyzing powers for the breakup reaction}

Breakup events collected for the two beam states with vector polarization, $\left(+\frac{2}{3}, 0\right),\left(-\frac{2}{3}, 0\right)$ and for an unpolarized beam were analyzed at a set of kinematical points, 


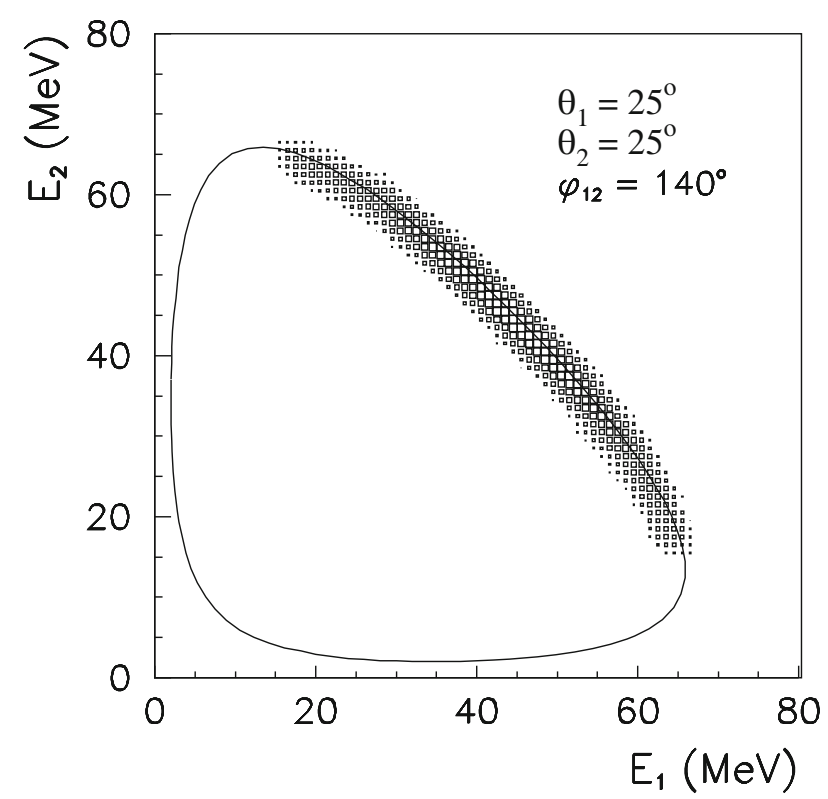

Fig. 6. An example of the kinematical spectrum $E_{2}$ versus $E_{1}$ for one selected angular configuration of the breakup process $\left(\theta_{1}=25^{\circ} \pm 2^{\circ}, \theta_{2}=25^{\circ} \pm 2^{\circ}, \varphi_{12}=140^{\circ} \pm 10^{\circ}\right)$. The line represents the kinematical curve calculated for the central values of the angular ranges.

defined by polar emission angles of the two protons, $\theta_{1}$, $\theta_{2}$, their relative azimuthal angle $\varphi_{12}$ and their energies, expressed by a variable $S$, defined below. Definitions of coordinate systems and angles are given in ref. [42]. For each configuration $\theta_{1}, \theta_{2}, \varphi_{12}$ of the two outgoing protons, the kinematical spectra $E_{2}$ versus $E_{1}$ were built (see an example in fig. 6). The kinematical energy relations are perfectly reproduced by the data, which confirms the reliability of the whole calibration procedure. The variable $S$ corresponds to the arc-length along the kinematical curve, with 0 chosen at the minimal value of $E_{2}$.

The obtained numbers of events have been normalized to the beam current collected in the Faraday Cup and corrected for losses due to the dead time. After normalization, the rates of events $N_{P}^{\xi}\left(N_{0}^{\xi}\right)$ for the selected polarization state $P$ (unpolarized beam) and a kinematical point $\xi=\left(\theta_{1}, \theta_{2}, \varphi_{12}, S\right)$ were obtained as a function of an angle $\phi$ (definition of an angle $\phi$ and overall convention as in ref. [64], see also ref. [42]). A ratio,

$$
f_{P}^{\xi}(\phi)=\frac{N_{P}^{\xi}(\phi)-N_{0}^{\xi}(\phi)}{N_{0}^{\xi}(\phi)},
$$

was constructed (in analogy to eq. (2)). Applying the formulae for the yield of the three-body breakup reaction induced by vector and tensor polarized spin-1 particles [64], $f_{P}^{\xi}(\phi)$ can be written in Cartesian coordinates as

$$
\begin{aligned}
f_{P}^{\xi}(\phi)= & -\frac{3}{2} \sin \phi P_{Z} A_{x}(\xi)+\frac{3}{2} \cos \phi P_{Z} A_{y}(\xi) \\
& +\frac{1}{2} \sin ^{2} \phi P_{Z Z} A_{x x}(\xi)+\frac{1}{2} \cos ^{2} \phi P_{Z Z} A_{y y}(\xi) \\
& -\frac{1}{2} \sin 2 \phi P_{Z Z} A_{x y}(\xi) .
\end{aligned}
$$

Parity conservation in strong interaction imposes certain relations between analyzing powers for mirror configurations, i.e. for the kinematical configurations differing only in the sign of the relative azimuthal angle $\varphi_{12}$. We introduce the notation $\xi \equiv\left(\tilde{\xi}, \varphi_{12}\right)$, where $\tilde{\xi}$ denotes all the kinematical variables but $\varphi_{12}$. The following combinations of asymmetries for mirror configurations $\left(\tilde{\xi}, \varphi_{12}\right)$ and $\left(\tilde{\xi},-\varphi_{12}\right)$ can be constructed:

$$
g_{P}^{\xi}(\phi)=\frac{f_{P}^{\tilde{\xi}, \varphi_{12}}(\phi)+f_{P}^{\tilde{\xi},-\varphi_{12}}(\phi)}{2}
$$

and

$$
h_{P}^{\xi}(\phi)=\frac{f_{P}^{\tilde{\xi}, \varphi_{12}}(\phi)-f_{P}^{\tilde{\xi},-\varphi_{12}}(\phi)}{2},
$$

which, when including indications resulting from the parity conservation rules [64], can be expressed as

$$
\begin{aligned}
g_{P}^{\xi}(\phi)= & \frac{3}{2} \cos \phi P_{Z} A_{y}(\xi) \\
& +\frac{1}{2} \sin ^{2} \phi P_{Z Z} A_{x x}(\xi)+\frac{1}{2} \cos ^{2} \phi P_{Z Z} A_{y y}(\xi)
\end{aligned}
$$

and

$$
h_{P}^{\xi}(\phi)=-\frac{3}{2} \sin \phi P_{Z} A_{x}(\xi)-\frac{1}{2} \sin 2 \phi P_{Z Z} A_{x y}(\xi) .
$$

Fitting the function (8) to the experimentally determined distribution $g_{P}^{\xi}(\phi)(6)$ leads to the determination of the parity-even analyzing powers, while the odd ones are obtained from the fit of the function (9) to the distribution $h_{P}^{\xi}(\phi)$ constructed according to eq. (7). Examples of such fits to the data are shown in fig. 7 . Values of the vector polarization $P_{Z}$ of the beam, extracted from the elastic scattering analysis (table 1 ), were used to extract the vector analyzing powers. By averaging the results for the two polarization states, statistically consistent with one another, the final values for $A_{x}$ and $A_{y}$ were obtained.

For an absolutely pure vector state, the ratios $g_{P}^{\xi}(\phi) / \cos \phi$ and $h_{P}^{\xi}(\phi) / \sin \phi$ reveal no $\phi$ dependence, and are reduced to constants: $\frac{3}{2} P_{Z} A_{y}(\xi)$ and $-\frac{3}{2} P_{Z} A_{x}(\xi)$, respectively. Such method of determining vector analyzing powers was applied, for control purposes, to the state $\left(-\frac{2}{3}, 0\right)$, which has a practically negligible tensor polarization component. Obtained values of $A_{x}$ and $A_{y}$ are consistent with the values resulting from the fits.

\subsection{Experimental uncertainties}

Experimental uncertainties of the vector analyzing powers vary strongly in the presented set of the data, but in all cases statistical uncertainties dominate. Their main contribution are statistical uncertainties of $N_{P}^{\xi}$ and $N_{0}^{\xi}$. In addition, these uncertainties comprise the statistical errors of the beam polarization $P_{Z}$ (cf. table 1$)$. In this paper 


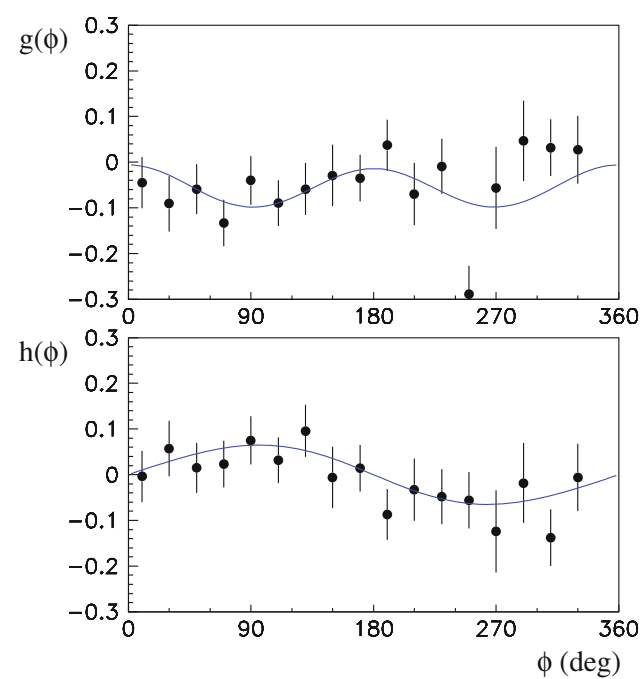

Fig. 7. Examples of asymmetry distributions $g_{P}^{\xi}(\phi)$ and $h_{P}^{\xi}(\phi)$ (see text), obtained for two (mirror) kinematical configurations with $\theta_{1}=25^{\circ}, \theta_{2}=15^{\circ}, \varphi_{12} \pm 80^{\circ}$ and $S=80 \mathrm{MeV}$, for $P=\left(-\frac{2}{3}, 0\right)$. The error bars represent statistical uncertainties. The lines result from the fit of the corresponding functions, as described in the text.

we take into account only the data points with statistical errors not exceeding 0.05 .

One of the main contributions to the systematic uncertainty of the vector analyzing powers arises from the systematic uncertainty of the vector polarization values. We attribute a relative error of about $5 \%$ to that effect. This uncertainty influences only the total scaling factor, common for all configurations and all $S$ values.

The data analysis presented in this paper relies on determination of the ratios of rates measured with polarized and unpolarized beams. Therefore, many experimental factors, like, e.g., the efficiencies of particle detection in the MWPC and in the scintillator hodoscope, losses due to hadronic interactions of protons, uncertainties in the determination of solid angles, cancel in the ratio (in the first order). The geometry of the setup is well known on the basis of the kinematics of the elastic scattering. Methods of using these well-defined kinematical relations for a precise determination of distances and of the beam position, described in ref. [39], were adapted from the SALAD system to the BINA Wall. As a result, a misalignment of about $1 \mathrm{~mm}$ between the beam axis and the detector center has been found and corrected. After that correction, it has been shown that, at the level below $0.5^{\circ}$, there was no systematic shift of the polar angles.

The uncertainty due to the contribution of accidental events can be neglected. The amount of such events can be determined on the basis of time spectra, containing groups of hits correlated in time with the trigger (true + random events, accepted in the analysis) and the ones originating from other beam bursts (random). Due to a very low beam current the contribution of random events is below $1 \%$, and its influence on the asymmetry values $f_{P}(\xi)$ is by an order of magnitude smaller than the statistical errors.
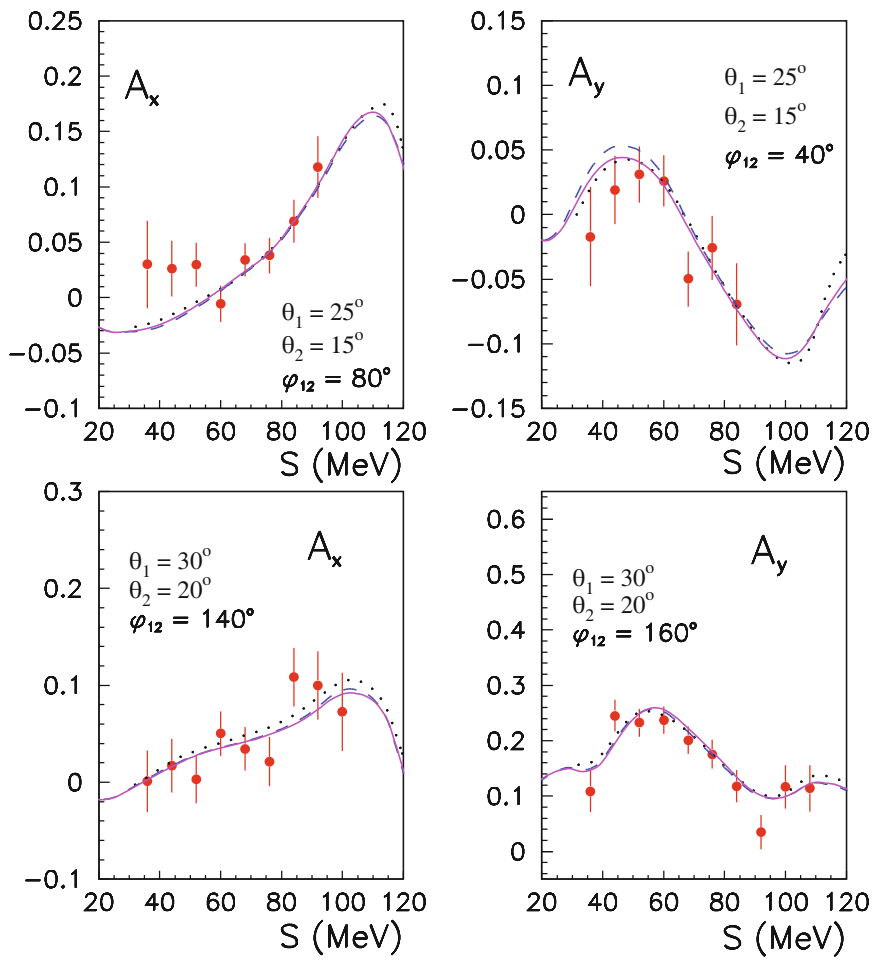

Fig. 8. Examples of the vector analyzing powers for the ${ }^{1} \mathrm{H}(\mathbf{d}, \mathrm{pp}) \mathrm{n}$ breakup reaction, obtained at configurations indicated in the panels. Error bars reflect statistical uncertainties. The results are compared to predictions obtained with the CDBonn potential only (dashed lines) and when TM99 3NF is included into the calculations (solid lines). Dotted lines represent CCP calculations with the Coulomb interaction included.

\section{Experimental results}

Vector analyzing powers $A_{x}(\xi)$ and $A_{y}(\xi)$ for the d-p breakup reaction at $100 \mathrm{MeV}$ have been obtained as a function of $S(\mathrm{MeV})$ for geometries defined by the emission angles of the outgoing protons. The selected geometries are spanned on a grid of polar angles $\theta_{1}, \theta_{2}$ between $15^{\circ}$ and $30^{\circ}$ in steps of $5^{\circ}$. The absolute values of the relative azimuthal angles $\varphi_{12}$ are changed between $0^{\circ}$ (for $\theta_{1} \neq \theta_{2}$ ) or $20^{\circ}$ (for $\theta_{1}=\theta_{2}$ ) and $180^{\circ}$, in steps of $20^{\circ}$. For each configuration with $\varphi_{12}$ from $20^{\circ}$ to $160^{\circ}$ its "mirror configuration", i.e. the configuration with a negative $\varphi_{12}$, has been analyzed. The angular ranges applied in the data analysis to define the kinematical configurations were wide enough to observe effects of averaging of the vector analyzing powers within these ranges. Therefore, in order to compare the data with the theoretical predictions, the same averaging had to be applied to the calculated values of the observables. Details of the procedure of averaging have been described in ref. [44].

In the whole studied range of the phase space, vector analyzing powers are very well reproduced by the theoretical calculations. In fig. 8 several examples of $A_{x}(\xi)$ and $A_{y}(\xi)$ distributions as a function of $S$ are shown. The data are compared to the results of three types of calculations: the ones based on the CD-Bonn potential, without and with TM99 3NF included, and CCP calculations (with 
Table 2. Global comparison of the whole set of the vector analyzing power data with the theoretical calculations.

\begin{tabular}{|l|c|c|}
\hline \multirow{2}{*}{ Theoretical approach } & \multicolumn{2}{|c|}{$\chi^{2} /$ d.o.f. } \\
\cline { 2 - 3 } & $A_{x}$ & $A_{y}$ \\
\hline CD-Bonn & 0.94 & 1.58 \\
CD-Bonn + TM99 & 0.94 & 1.58 \\
CCP (CD-Bonn+ + +Coulomb) & 0.92 & 1.56 \\
\hline
\end{tabular}

explicit $\Delta$ ) taking into account Coulomb interaction between protons. For the presented configurations, as well as for the whole data set, the predicted effects of $3 \mathrm{NF}$ and of the Coulomb force are practically negligible and the data description by the NN potential alone is very good. Quantitatively, the comparison of the whole data set (about 400 data points per observable) was performed by means of $\chi^{2} /$ d.o.f. Values of the global $\chi^{2} /$ d.o.f. are shown in table 2 , confirming insensitivity of the results to the theoretical approach applied. The $\chi^{2} /$ d.o.f. values obtained for $A_{y}(\xi)$ are slightly higher than the ones for $A_{x}(\xi)$, which is consistent with the results obtained at the beam energy of $130 \mathrm{MeV}$ and very forward angles [43].

The energy of the relative motion of the two outgoing protons $\left(E_{r e l}\right)$ turned out to be an important kinematical variable pointing to various effects in the cross sections of the d-p breakup at the beam energy of $130 \mathrm{MeV}$, as presented in refs. $[41,52]$. Also certain problems with reproducing the tensor analyzing powers $A_{x x}$ and $A_{y y}$ at this energy seem to be localized in the region of small $E_{r e l}$ [42]. Therefore, dependencies of $\chi^{2} /$ d.o.f. on $E_{r e l}$ have also been investigated and are presented in fig. 9. Again, no particular trend can be observed and a good description of the data is confirmed.

\section{Summary and conclusions}

A large set of experimental data for the vector analyzing powers has been obtained for deuteron-proton collisions at the deuteron beam energy of $100 \mathrm{MeV}$. It comprises nearly complete angular distribution for the vector analyzing power $i T_{11}^{e l}$ of the deuteron-proton elastic scattering, as well as the vector analyzing powers $A_{x}(\xi)$ and $A_{y}(\xi)$ for the ${ }^{1} \mathrm{H}(\mathbf{d}, \mathrm{pp}) \mathrm{n}$ breakup reaction obtained on a systematic grid of laboratory angles covering substantial part of the phase space. These observables turned out to be practically insensitive to aspects of the nuclear dynamics beyond the pure NN interaction. The theoretical calculations limited to the pairwise NN interaction modelled with the CD-Bonn potential describe the whole data set very well. The conclusions obtained at $100 \mathrm{MeV}$ are very similar to the outcome of the earlier studies at the beam energy of $130 \mathrm{MeV}[26,42,43]$.

The presented here investigations will be in future extended to geometries with one of protons registered in the Ball part of the detector. Moreover, the tensor analyzing powers and the differential cross section will be

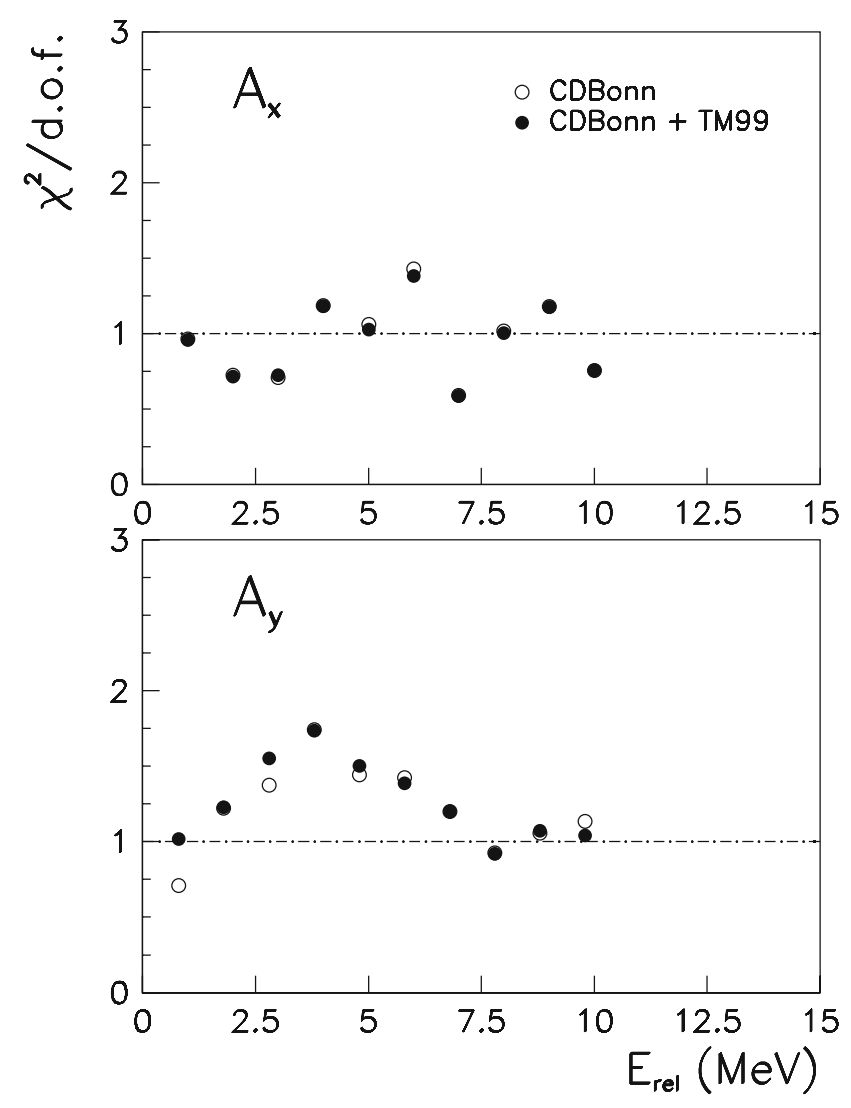

Fig. 9. Quality of the description of the vector analyzing powers given by various models, presented as $\chi^{2}$ per degree of freedom, as a function of the energy of the relative motion of the two breakup protons. CCP results with the Coulomb included are practically identical, therefore they are not shown for clarity of the picture.

determined. For these observables a significant influence of a Coulomb force is expected, particularly strong in the case of the differential cross section. Therefore that part of dynamics can be studied in detail and, confronting the results with the outcome of the experiment at $130 \mathrm{MeV}$, the Coulomb force effects can be investigated in a function of beam energy.

This work was supported by the European Commission within the Sixth Framework Programme through I3-EURONS (contract no. RII3-CT-2004-506065) and by the Polish 2009-2011 science founds as a research project No. N202 034836. One of the authors (ES) would like to thank "Nederlandse Organisatie voor Wetenschappelijk Onderzoek" (NWO) for the fellowship at KVI, which was of vital importance for the preparation and for performing of the experiment. We thank the KVI staff for the hospitality and for granting us the high-quality polarized beam and excellent experimental conditions. The numerical calculations were in part performed on the supercomputer cluster of the JIC, Jülich, Germany. The work was also partially supported by the Polish National Center under Grant No. DEC-2011/01/B/ST2/00578. 
Open Access This is an open access article distributed under the terms of the Creative Commons Attribution License (http://creativecommons.org/licenses/by/3.0), which permits unrestricted use, distribution, and reproduction in any medium, provided the original work is properly cited.

\section{References}

1. R. Lazauskas et al., Phys. Rev. C 71, 034004 (2005).

2. A. Deltuva, A.C. Fonseca, Phys. Rev. C 67, 021001 (2007).

3. V.G.J. Stoks, R.A.M. Klomp, M.C.M. Rentmeester, J.J. de Swart, Phys. Rev. C 48, 792 (1993).

4. E. Epelbaum, H.-W. Hammer, U.-G. Meissner, Rev. Mod. Phys. 81, 1773 (2009).

5. J. Fujita, H. Miyazawa, Prog. Theor. Phys. 17, 360 (1957).

6. S.A. Coon, H.K. Han, Few-Body Syst. 30, 131 (2001).

7. H.T. Coelho et al., Phys. Rev. C 28, 1912 (1983).

8. B.S. Pudliner, V.R. Pandharipande, J. Carlson, S.C. Pieper, R.B. Wiringa, Phys. Rev. C 56, 1720 (1997).

9. S.C. Pieper, V.R. Pandharipande, R.B. Wiringa, J. Carlson, Phys. Rev. C 64, 014001 (2001).

10. A. Nogga et al., Phys. Rev. C 67, 034004 (2003).

11. W. Glöckle et al., Phys. Rep. 274, 107 (1996) and references therein.

12. H. Witala, W. Glöckle, D. Huber, J. Golak, H. Kamada, Phys. Rev. Lett. 81, 1183 (1998).

13. H. Sakai et al., Phys. Rev. Lett. 84, 5288 (2000).

14. R. Bieber et al., Phys. Rev. Lett. 84, 606 (2000).

15. K. Hatanaka et al., Phys. Rev. C 66, 044002 (2002).

16. P. Mermod et al., Phys. Lett. B 597, 243 (2004).

17. K. Sekiguchi et al., Phys. Rev. C 70, 014001 (2004).

18. K. Sekiguchi et al., Phys. Rev. Lett. 95, 162301 (2005).

19. K. Ermisch et al., Phys. Rev. Lett. 86, 5862 (2001).

20. K. Ermisch et al., Phys. Rev. C 68, 051001 (2003).

21. K. Ermisch et al., Phys. Rev. C 71, 064004 (2005).

22. B. von Przewoski et al., Phys. Rev. C 74, 064003 (2006).

23. Y. Maeda et al., Phys. Rev. C 76, 014004 (2007).

24. H.R. Amir-Ahmadi et al., Phys. Rev. C 75, 041001(R) (2007).

25. H. Mardanpour et al., Eur. Phys. J. A 31, 383 (2007).

26. E. Stephan et al., Phys. Rev. C 76, 57001 (2007).

27. A. Ramazani-Moghaddam-Arani et al., Phys. Rev. C 78, 014006 (2008).

28. A. Deltuva, K. Chmielewski, P.U. Sauer, Phys. Rev. C 67, 034001 (2003).

29. A. Deltuva, R. Machleidt, P.U. Sauer, Phys. Rev. C 68, 024005 (2003).
30. A. Deltuva, A.C. Fonseca, P.U. Sauer, Phys. Rev. C 72, 054004 (2005).

31. A. Deltuva, A.C. Fonseca, P.U. Sauer, Phys. Rev. C 73 , 057001 (2006).

32. E. Epelbaum, Prog. Part. Nucl. Phys. 57, 654 (2006).

33. St. Kistryn et al., Nucl. Phys. A 548, 49 (1992).

34. J. Balewski et al., Nucl. Phys. A 581, 131 (1995).

35. M. Allet et al., Phys. Rev. C 50, 602 (1994).

36. M. Allet et al., Phys. Lett. B 376, 255 (1996).

37. J. Zejma et al., Phys. Rev. C 55, 42 (1997).

38. K. Bodek et al., Few-Body Syst. 30, 65 (2001).

39. St. Kistryn et al., Phys. Rev. C 68, 054004 (2003).

40. St. Kistryn et al., Phys. Rev. C 72, 044006 (2005).

41. St. Kistryn et al., Phys. Lett. B 641, 23 (2006).

42. E. Stephan et al., Phys. Rev. C 82, 014003 (2010).

43. I. Ciepal et al., Phys. Rev. C 85, 017001 (2012).

44. E. Stephan et al., Eur. Phys. J. A 42, 13 (2009).

45. M. Eslami-Kalantari et al., Mod. Phys. Lett. A 24, 839 (2009).

46. H. Mardanpour et al., Phys. Lett. B 687, 149 (2010).

47. H.O. Meyer et al., Phys. Rev. Lett. 93, 112502 (2004).

48. K. Sekiguchi et al., Phys. Rev. C 79, 054008 (2009).

49. S. Kuroita et al., Few-Body Sys. 44, 53 (2008).

50. S. Kuroita et al., Few-Body Sys. 50, 287 (2011).

51. K. Sagara, Few-Body Sys. 48, 59 (2010).

52. N. Kalantar-Nayestanaki, E. Epelbaum, J.G. Meschendorp, A. Nogga, Rep. Prog. Phys. 75, 016301 (2012).

53. H.R. Kremers, A.G. Drentje, AIP Conf. Proc. 421, 502 (1997).

54. N. Kalantar-Nayestanaki, J. Mulder, J. Zijlstra, Nucl. Instrum. Methods A 417, 215 (1998).

55. N. Kalantar-Nayestanaki et al., Nucl. Instrum. Methods A 444, 591 (2000).

56. M. Volkerts, A. Bakker, N. Kalantar-Nayestanaki, H. Fraiquin, A. Eads, T. Rinckel, K. Solberg, Nucl. Instrum. Methods Phys. Res. A 428, 432 (1999).

57. St. Kistryn, Readout System for Medium-Size Experiments, in Advanced Technologies (Sciyo, Rijeka, 2010) ISBN 978-953-7619.

58. H.G. Essel, N. Kurz, IEEE Trans. Nucl. Sci. 47, 337 (2000).

59. J.B. Birks, Proc. Phys. Soc. A 64, 874 (1951).

60. C.N. Chou, Phys. Rev. 87, 904 (1952).

61. G.G. Ohlsen, Rep. Prog. Phys. 35, 717 (1972).

62. H. Witała et al., Few-Body Sys. 15, 67 (1993).

63. R. Sworst, PhD thesis, Jagiellonian University, 2009.

64. G.G. Ohlsen, R.E. Brown, F.D. Correll, R.A. Hardekopf, Nucl. Instrum. Methods 179, 283 (1981). 